\title{
3 Gene-tree reconciliation with MUL-trees to resolve polyploidy events
}

4

5 Gregg W.C. Thomas*, S. Hussain Ather, and Matthew W. Hahn

7 Department of Biology and School of Informatics and Computing, Indiana University,

8 Bloomington, IN 47405.

10 *Corresponding author

11 Email: grthomas@indiana.edu 


\section{Abstract}

Polyploidy can have a huge impact on the evolution of species, and it is a common occurrence, especially in plants. The two types of polyploids - autopolyploids and allopolyploids - differ in the level of divergence between the genes that are brought together in the new polyploid lineage. Because allopolyploids are formed via hybridization, the homoeologous copies of genes within them are at least as divergent as orthologs in the parental species that came together to form them. This means that common methods for estimating the parental lineages of allopolyploidy events are not accurate, and can lead to incorrect inferences about the number of gene duplications and losses. Here, we have adapted an algorithm for topology-based gene-tree reconciliation to work with multi-labeled trees (MUL-trees). By definition, MUL-trees have some tips with identical labels, which makes them a natural representation of the genomes of polyploids. Using this new reconciliation algorithm we can: accurately place allopolyploidy events on a phylogeny, identify the parental lineages that hybridized to form allopolyploids, distinguish between allo-, auto-, and (in most cases) no polyploidy, and correctly count the number of duplications and losses in a set of gene trees. We validate our method using gene trees simulated with and without polyploidy, and revisit the history of polyploidy in data from the clades including both baker's yeast and bread wheat. Our re-analysis of the yeast data confirms the allopolyploid origin and parental lineages previously identified for this group. The method presented here should find wide use in the growing number of genomes from species with a history of polyploidy. 
Polyploidy as a result of whole genome duplication (WGD) can be a key evolutionary

event. At least two ancient WGDs have been postulated at the origin of vertebrate animals (Ohno

(Amores et al. 1998; Van de Peer 2004). Polyploidy events are far more common in plants. It is combined with the success of flowering plants suggests that WGDs must confer some advantage, possibly by increasing speciation rates (Werth and Windham 1991; Lynch and Force 2000; but see Mayrose et al. 2011; Muir and Hahn 2015), by decreasing extinction rates (Crow and Wagner 2006), or by providing species with a large amount of genetic material from which novel

47 phenotypes can arise (Adams and Wendel 2005; Soltis and Soltis 2009; Edger et al. 2015).

Because of their importance in adaptation and speciation, multiple methods have been employed to study polyploidy events. The goals of these methods vary and can include: identifying polyploidy events, placing events in a phylogenetic context to identify the lineages on

51 which they took place, or counting gene duplications and losses in the presence of polyploidy

52 (Table 1). When placing polyploidy events in a phylogenetic context, either relatively or absolutely in time, care must be taken to distinguish between the two types of polyploidy.

54 Autopolyploidy occurs when an individual inherits sets of chromosomes from parents of the same species. Genes duplicated as the result of autopolyploidy are paralogous. Following Glover et al. (2016), we will refer to these as ohnologs, although this term was originally used to 


\section{Outcomes}

\begin{tabular}{|c|c|c|c|c|}
\hline & $\begin{array}{l}\text { Identify } \\
\text { polyploidy } \\
\text { events }\end{array}$ & $\begin{array}{l}\text { Place polyploidy } \\
\text { on phylogeny }\end{array}$ & $\begin{array}{l}\text { Identify parental } \\
\text { lineages of } \\
\text { allopolyploids }\end{array}$ & $\begin{array}{l}\text { Count } \\
\text { duplications } \\
\text { and losses }\end{array}$ \\
\hline $\begin{array}{l}\text { Low-copy gene } \\
\text { trees/networks } \uparrow\end{array}$ & & + & + & \\
\hline $\mathrm{K}_{\mathrm{s}}$ peaks & + & $+*$ & & \\
\hline $\begin{array}{l}\text { LCA reconciliation } \\
\text { to standard trees } \dagger\end{array}$ & + & $+^{*}$ & & $+^{*}$ \\
\hline Count-based $t$ & + & + & & + \\
\hline $\begin{array}{l}\text { LCA reconciliation to } \\
\text { MUL-trees } \dagger \S\end{array}$ & + & + & + & + \\
\hline
\end{tabular}

*These inferences are often incorrect for allopolyploids. See main text.

$\dagger$ Uses topology

† Uses branch lengths

61

$\S$ Method described in this paper.

64 an individual inherits sets of chromosomes from parents of different species through

65 hybridization. Genes duplicated as the result of allopolyploidy are called homoeologs. The term

66 "homoeolog" was originally applied to relationships between chromosomes in allopolyploids

67 (Glover et al. 2016), and seems most appropriate as a descriptor of the genealogical relationships

68 of homologous genes within allopolyploids: not quite orthologs and not quite paralogs (Glover et

69 al. 2016). Recognizing that homoeologs have relationships differing from those occurring

70 between homologous genes within autopolyploids is key in preventing the mis-classification of

71 allopolyploids as autopolyploids (cf. Doyle and Egan 2010). 
There are many different methods used to identify and study polyploidy events. Non-

73

74

75

76

77

78

79

80

81

82

phylogenetic methods, such as synteny and karyotyping, have long been used to identify

polyploid species and will not be discussed further here (for review see Barker et al. 2015;

Kellogg 2016). Phylogenetic methods (Table 1) use the relationships of genes between closely related species to identify and place polyploidy events on a phylogeny. One common approach is

to build phylogenies of low-copy genes and to treat the resulting gene trees as species

relationships (see Table S1 for a summary of studies that have used each method). Because these

are low-copy genes, only polyploid species should be represented with multiple copies, in which case each copy represents either an ohnolog or a homoeolog. If the polyploid species or clade in question is an autopolyploid, the ohnologs will be sister to each other in the genome tree (topology in Fig. 1a); if they are allopolyploids, the homoeologs will be sister to different diploid taxa (as long as these lineages are sampled; topology in Fig 1b). These diploid taxa are either the direct progenitors of the allopolyploid species (the parental species) or the extant taxa most closely related to the progenitors (the parental lineages; Fig S1) if the direct progenitors are extinct or were not sampled. Such methods can benefit from including more species closely related to the parental lineages of the polyploids in order to separate multiple WGDs close in time. Incongruence caused by error in gene tree inference or incomplete lineage sorting can be addressed by sequencing more genes (e.g. Brassac and Blattner 2015), or by comparing nuclear loci to genes in the mitochondria or chloroplast (e.g. Popp and Oxelman 2001), and methods have been devised to make consensus phylogenies from sets of gene trees (e.g. Huber et al. 2006; Marcussen et al. 2012; Marcussen et al. 2015). However, the use of low-copy genes is only applicable for more recent polyploidy events, as additional paralogs created by gene duplication 
94 (and the loss of homoeologs) are not considered by these methods (Marcussen et al. 2012; Jones

95 et al. 2013).

In order to identify previously unknown polyploidy events, $\mathrm{K}_{\mathrm{s}}$-based, least common

97 ancestor (LCA) reconciliation, and count-based methods are used (Table 1; Table $\mathrm{S} 1$ ). $\mathrm{K}_{\mathrm{s}}$-based methods start by identifying pairs of duplicate genes within a species of interest, then measuring the synonymous divergence, or $\mathrm{K}_{\mathrm{s}}$, between them. In a species that has not experienced polyploidy, the expectation is that most duplicates will be very recent - and have a low $\mathrm{K}_{\mathrm{s}}-$ while very few pairs will have high $\mathrm{K}_{\mathrm{s}}$ (Lynch and Conery 2000). However, in a lineage in which polyploidy has occurred, peaks observed in this distribution are said to correspond to a burst of duplications from the WGD, though this is not always the case (see below; Lynch and Conery 2000; Blanc and Wolfe 2004). Peaks observed in a single species are placed on the tip branch of a species phylogeny, while peaks of $\mathrm{K}_{\mathrm{s}}$ shared among multiple species are placed on internal branches (e.g. Cui et al. 2006; Barker et al. 2008; Barker et al. 2009). events onto an accepted species phylogeny via gene tree-species tree reconciliation (Goodman et al 1979; Page 1994). Gene duplications on each branch of a species tree can be counted, and branches with an unusually large number of gene duplications per unit time can indicate a

111 polyploidy event (Cannon et al. 2015). Often LCA reconciliation is carried out on simplified

112 gene trees (Bowers et al. 2003; Li et al. 2015), but the inferences remain the same. Finally, 113 recent likelihood-based "count" methods use gene copy-numbers to identify branches with more 114 gene duplications than expected without polyploidy (Rabier et al. 2014; Tiley et al. 2016).

115 Neither gene trees nor pairwise distances are calculated among homologs, and instead the 116 number of gene copies in each genome is used as a character that evolves across a species 
117 phylogeny. $\mathrm{K}_{\mathrm{s}}$, LCA, and count-based methods can be used for analysis of polyploidy events at 118 any depth in time, though they are most commonly used for more ancient events where one or 119 both parental lineages are extinct.

However, the $\mathrm{K}_{\mathrm{s}}$ and LCA methods described above do not give a full picture of the

121 evolutionary history of a polyploidy event, and may be positively misleading about multiple

122 aspects of WGDs. This is because these methods do not take into account the difference in the

123 degree of divergence between duplicate gene pairs in the two types of polyploidy. Specifically,

$124 \mathrm{~K}_{\mathrm{s}^{-}}$and LCA-based methods fail because they treat the two homologous copies of a gene arising

125 from polyploidy as paralogs regardless of their mode of origin - that is, homologous genes

126 related by a duplication event at their most recent common ancestor (Fitch 1970). However, for

127 allopolyploids, these two genes are more akin to orthologs, as they are actually related by a

128 speciation event at their most recent common ancestor (Glover et al. 2016). While these

129 methods will work for autopolyploids - since the divergence time of ohnologs is the true timing

130 of the WGD (Fig. 1a) - for allopolyploids they incorrectly identify the most recent common

131 ancestor of the species that hybridized to form the polyploid as the time that the WGD occurred

132 (Fig. 1b) (Doyle and Egan 2010; Kellogg 2016). When using LCA-based gene tree methods,

133 WGDs due to allopolyploidy can also lead to incorrect inferences of many additional gene

134 duplications and losses when none have occurred (see Methods). 

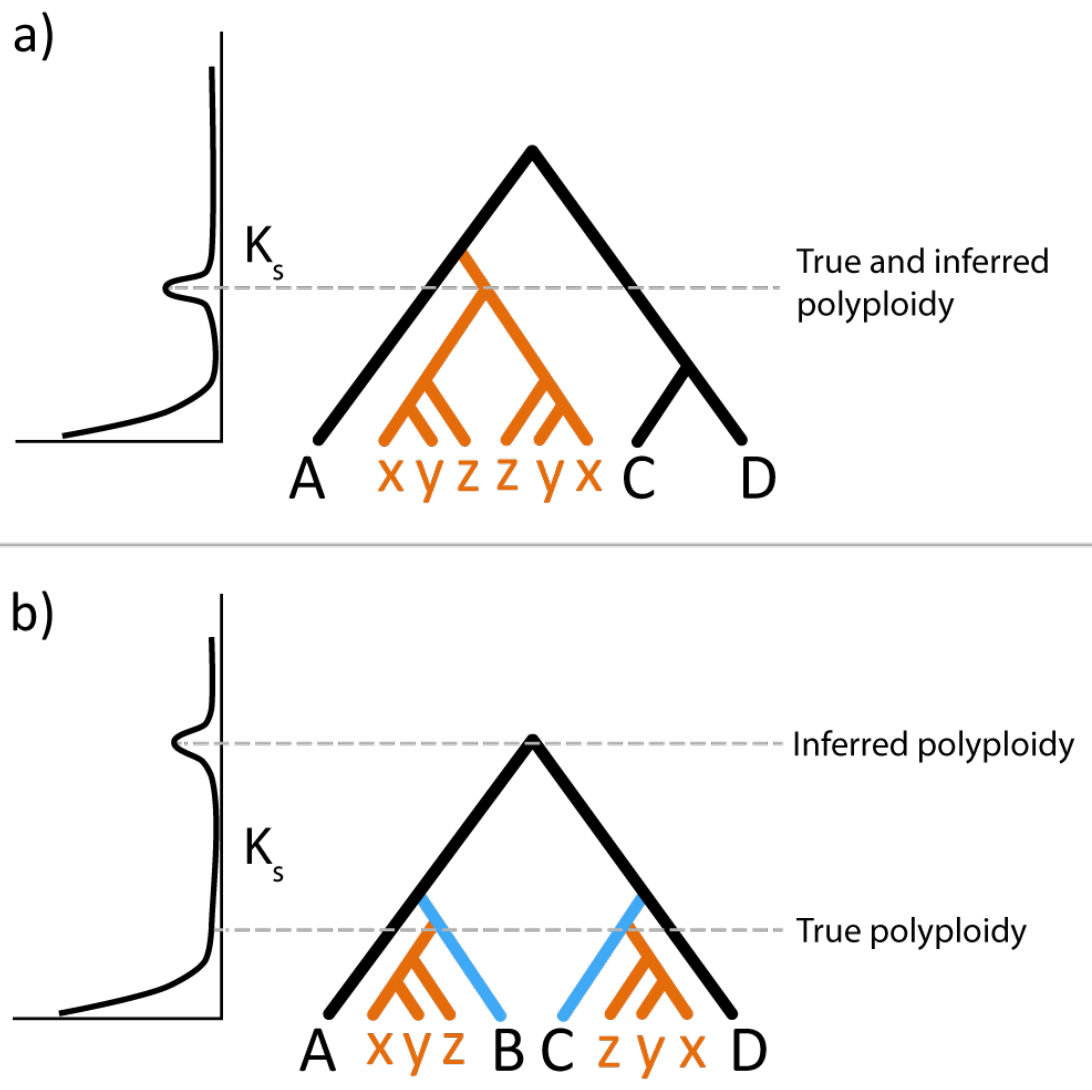

One major issue shared by $\mathrm{K}_{\mathrm{s}}$-based, LCA, and gene count methods when used to study polyploidy is that the typical bifurcating, singly-labeled representations of species relationships

144 (henceforth referred to as singly-labeled trees) only represent one of the multiple homoeologous sub-genomes (sets of chromosomes) in any allopolyploid species (Fig. 2a). Species networks are the correct representation for allopolyploids as they can highlight both the parental lineages

147 involved and the relative timing of the hybridization event (Fig. 2b) (Linder and Rieseberg 2004;

148 Huber and Moultan 2006; Jones et al. 2013). However, networks represent species relationships, 149 and therefore can be less practically useful for analyses involving multiple individual genes in 150 allopolyploid genomes. An alternative and fully equivalent representation to the species 
151 relationships in networks uses multi-labeled trees (MUL-trees, sometimes referred to as "genome

152 trees" in this context) to represent genome relationships for polyploidy events (Fig. 2c) (Huber et

153 al 2006; Lott et al. 2009). MUL-trees are trees in which the tip labels are not necessarily unique

154 (Huson et al 2006); this allows one to represent all sub-genomes in an allopolyploid as

155 descendants of different parental lineages (Fig. 2c), or as descendants of the same lineage for

156 autopolyploids (e.g. Fig. 1a).

\section{Figure 2:}

158

a)

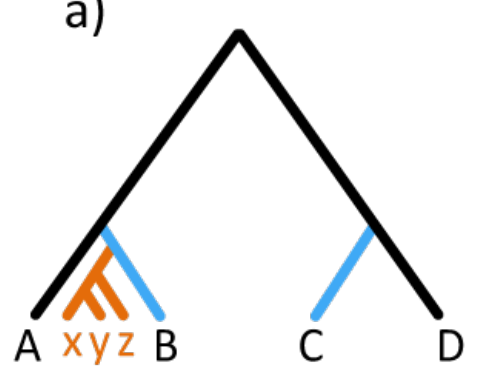

b)

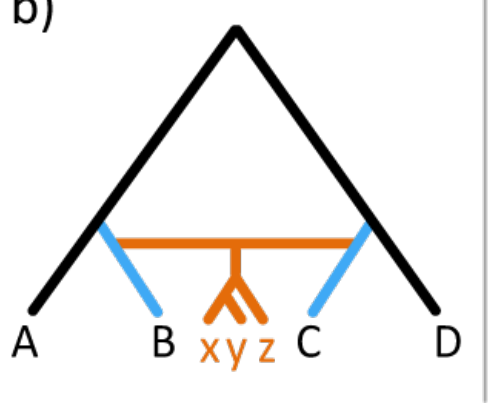

c)

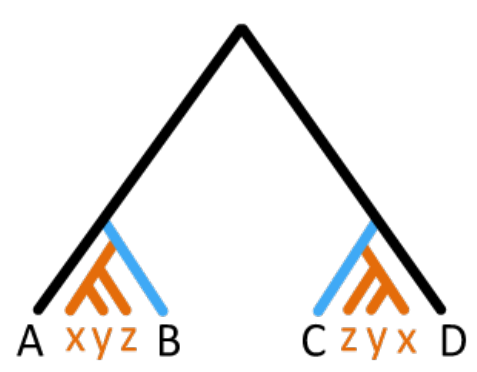

Here, we have adapted the LCA algorithm for use with MUL-trees, implementing this method in the software package GRAMPA (Gene-tree Reconciliation Algorithm with MUL-trees for Polyploid Analysis). This representation and algorithm allows us to correctly infer gene duplications and losses in the presence of polyploidy, and to identify the most likely placement of polyploid clades and their parental lineages. In most cases, it should also be able to infer whether or not a polyploidy event has taken place. We demonstrate that this new method works on simulated data, and we revisit two different datasets that include allopolyploid species, confirming a newly presented conclusion on the parental lineages leading to the clade that 167 includes baker's yeast. 


\section{Methods}

\section{Algorithm}

While the problem of reconciling gene trees to reticulated phylogenies has been explored

173 before (Yu et al. 2013; To and Scornavacca 2015), we have devised an LCA mapping algorithm

174 that reconciles gene phylogenies to genome relationships represented as MUL-trees, a natural

175 representation of polyploidy events. The LCA mapping algorithm is a method that identifies and counts duplication and loss events on a gene tree given an accepted singly-labeled species tree

177 (Goodman et al 1979; Page 1994). It can also be used for species tree inference by searching for the species tree that minimizes the total number of duplications and losses inferred given a set of gene trees (Guigó et al. 1996). The main hurdle in applying LCA mapping to MUL-trees is that, when reconciling to a MUL-tree, some nodes have more than one possible map. In particular, some tip nodes cannot be initialized with a single map (because tips are necessarily not uniquely labeled), which subsequently allows internal nodes to also have more than one possible map. We side-step this problem by trying all possible combinations of initial tip maps and applying the parsimony assumption - that the correct map will have the lowest score. species at the tips below it in the tree. The same node is also associated with a node in the species tree, $n_{s}$, through a map, $M\left(n_{g}\right) . M\left(n_{g}\right)=n_{s}$, where $n_{s}$ is the node in the species tree that is the least common ancestor of the species that define $n_{g}$. For example, in the gene tree

189 depicted in Figure 3a, node $1 \mathrm{G}$ is defined by the tips A1 and B1. These tips map to species A and $\mathrm{B}$, respectively, and the first node in the singly-labeled species tree (going from tips to root) that 
191 includes species A and B is $1 \mathrm{~S}$ (Fig. 3b). Therefore, $M(1 G)=1 S$. This process is repeated for

192 every internal node in the gene tree until all nodes are mapped. For each $n_{g}$ there is a single

193 possible node in the species tree to which it can map; however, nodes in the species tree can have

194 multiple nodes map to them. Nodes in the gene tree are said to be duplication nodes when they

195 map to the same species tree node as at least one of their descendants (for example, nodes $3 \mathrm{G}$

196 and 4G in Fig. 3b).

When the mapping of a gene tree $\left(T_{G}\right)$ is performed, nodes in the gene tree are classified

$$
l_{b_{g}}=\left(\operatorname{depth}\left(M\left(n_{g}\right)\right)-\operatorname{depth}\left(M\left(\operatorname{ancestor}\left(n_{g}\right)\right)\right)-1\right)+\operatorname{IsDup}\left(\operatorname{ancestor}\left(n_{g}\right)\right)
$$

202 Where IsDup is a function that returns 1 if the ancestor of $n_{g}$ is a duplication node and 0 score $\left(S_{T_{G}}\right)$ for this gene tree is:

$$
S_{T_{G}}=D_{T_{G}}+L_{T_{G}}
$$


bioRxiv preprint doi: https://doi.org/10.1101/058149; this version posted March 21, 2017. The copyright holder for this preprint (which was not certified by peer review) is the author/funder, who has granted bioRxiv a license to display the preprint in perpetuity. It is made available under aCC-BY-NC-ND 4.0 International license.

\section{Figure 3:}

a)

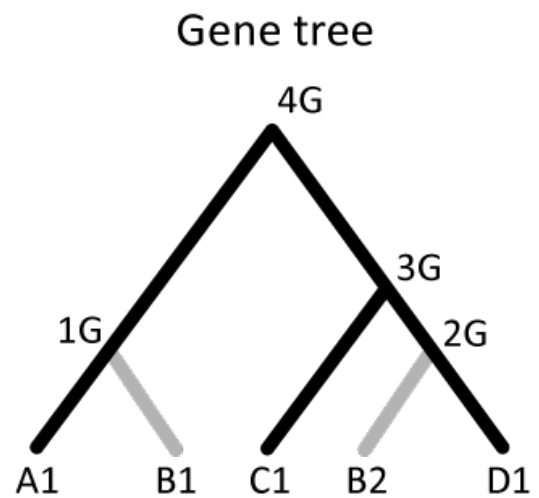

b)
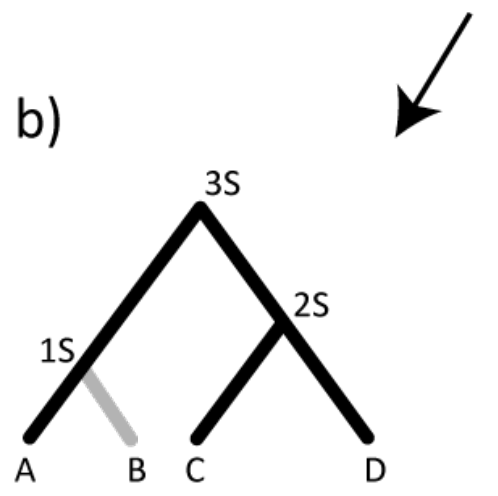

\begin{tabular}{|ll|}
\hline Node & Maps to \\
$1 G$ & $1 S$ \\
$2 G$ & $3 S$ \\
$3 G$ & $3 S$ \\
$4 G$ & $3 S$ \\
\hline
\end{tabular}

Reconciliation to singly-labeled species tree Score $=2$ duplications +5 losses

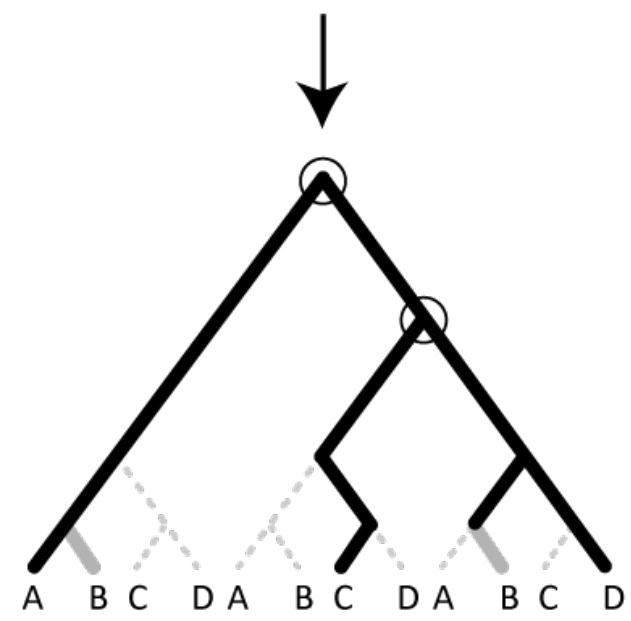

Reconciled gene tree
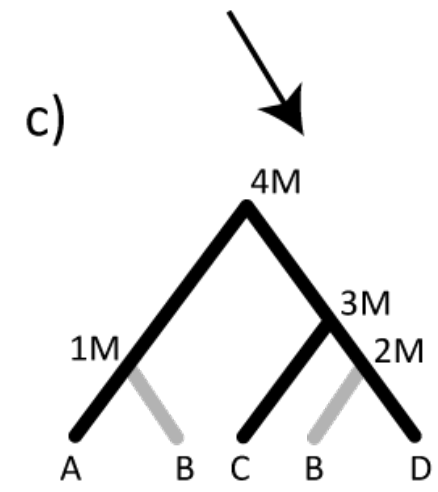

Node Maps to $1 \mathrm{G} \quad 1 \mathrm{M}$

$2 \mathrm{G} \quad 2 \mathrm{M}$

$3 \mathrm{G} 3 \mathrm{M}$

4G $4 \mathrm{M}$

Reconciliation to MUL-tree Score $=0$ duplications +0 losses 

does not map to the root of the species tree. This happens because there are no nodes in the gene

215

216

217

218

tree mapping to entire branches of the species tree, and therefore no calculations can be

performed. In these cases (when $n_{g}=$ root) we add $\operatorname{depth}\left(M\left(n_{g}\right)-1\right)$ to $L_{T_{G}}$ because the most parsimonious solution is simply a single loss of each branch above this node in the species tree.

The entire reconciliation process hinges on the fact that the mapping function is initialized with the tips of the gene tree mapped to their corresponding species label in the species tree. In a MUL-tree, repeated clades represent the sub-genomes of the polyploid species, and their placement in the MUL-tree defines parental lineages of the polyploid event (Fig. 2c, Fig S1). Given a gene tree, we proceed with the LCA mapping algorithm as described above, except that any tip that maps to a polyploid species now has two possible initial maps: to either of the sub-genomes represented in the MUL-tree (species "B" in Fig. 3c). This leads to unresolved internal maps and the inability to classify nodes correctly. To solve this problem, we run LCA mapping with a tip within a polyploid clade initialized to one sub-genome first, and then we run LCA mapping again with that same tip initialized to the other sub-genome, giving us two maps and two reconciliation scores for the single gene tree. We then apply the parsimony principle for these two possible maps: whichever initial mapping results in the lowest score is the correct map. If there is more than one gene in the gene tree from the polyploid clade we try all possible combinations of initial maps. In cases where multiple different mappings are all tied for the lowest score, we report all possible mappings.

The MUL-tree reconciliation algorithm is applicable for any number of genes in any number of polyploid species, but the algorithm becomes very slow for large polyploid clades 
236 because of the large number of combinations of initial maps to consider. Given that a gene tree

237 has $m$ genes represented from polyploid species, the run time for mapping this gene tree is

$O\left(2^{m} n\right)$, since the mapping algorithm itself is linear for a tree with $n$ nodes (Zmasek and Eddy 2001) and we perform $2^{m}$ maps (Fig. S2b). A similar brute force method was devised by Yu et al. (2013) when mapping alleles in a gene tree to a species tree in order to infer hybridization. In the context of polyploidy, we devised several methods to expedite the process of choosing the

242 correct map by using context within both the gene tree and the MUL-tree. If any genes from

243 different polyploid species form a clade within the gene tree, then the most parsimonious

244 solution will always have them mapping to the same sub-genome in the MUL-tree. We group

245 these nodes together, essentially treating the clade as a single tip of the tree, and try both maps on

246 the group as a whole rather than on each tip individually (Fig. S2c). We also consider the species

247 sister to the polyploid clades in the MUL-tree. If we observe clades in the gene tree that include

248 only polyploid species and these sister species, then the most parsimonious initial map for that

249 group is guaranteed to be the one in the MUL-tree with the corresponding sister species. The

250 algorithm therefore fixes the maps of these nodes before proceeding (Fig. S2d). This method

251 gives a reconciliation score for mapping a single gene tree to a single MUL-tree, and can be

252 applied to a set of gene trees to obtain a total reconciliation score for a MUL-tree. Even with the

253 speed-ups described above, some gene trees can still take an exorbitant amount of time to

254 reconcile. We therefore place a cap of 15 on the number of groups that can be considered for a

255 given gene tree. This limits any gene tree to at most $2^{15}=32,768$ possible maps. Gene trees

256 over this cap are skipped. The number of groups in a gene tree can vary for different MUL-trees.

257 To ensure consistency when comparing scores between MUL-trees, we first calculate the number 
258 of groups for each gene tree/MUL-tree combination and filter out gene trees that are over the cap

259 for any MUL-tree.

Thus far we have assumed that the placement of the polyploidy event is already known, since a single MUL-tree represents a single polyploid scenario. When it is not known, we have implemented a search strategy to find the most parsimonious placement of a polyploidy event, given a singly-labeled species tree. We define two nodes of interest in a singly-labeled species tree that we use to build a MUL-tree. Node H1 defines the location of the sub-genome for the

265 polyploid species represented in the singly-labeled species tree (as in Fig. S3a). Node H2 defines

266 the location of the second parental lineage and unrepresented polyploid sub-genome (Fig. S3a).

267 When $\mathrm{H} 1$ is specified, the sub-tree that is rooted by it and the branch that it subtends are copied and placed on the branch that is subtended by H2. Our modified LCA mapping algorithm is then run on the resulting MUL-tree, and a total reconciliation score is obtained by summing across scores for all gene trees. The algorithm can be limited to a specified pair of $\mathrm{H} 1$ and $\mathrm{H} 2$ nodes, or

271 only a specified H1 node (searching for H2), or no nodes specified (searching for both H1 and

$272 \mathrm{H} 2$ ). The MUL-tree defined by a particular $\mathrm{H} 1$ and $\mathrm{H} 2$ with the lowest total reconciliation score 273 reveals the location and type of the most parsimonious polyploidy event. Placement of H1 and $274 \mathrm{H} 2$ as sister to different lineages indicates allopolyploidy, while placement of $\mathrm{H} 1$ and $\mathrm{H} 2$ on the 275 same node in the species tree represents an autopolyploid event. We also perform LCA reconciliation to the input singly-labeled tree. In many instances, if no polyploidy has occurred in

277 the sampled lineages, the singly-labeled tree will return the lowest score. auto-, and no polyploidy in most cases (see Results). However, there are circumstances in which 
281 infer autopolyploidy. The distinction between parental lineages and parental species is important

282 when discussing allopolyploids. We define a parental lineage of an allopolyploid as the clade of

283 species more closely related to one sub-genome than the other (Fig. S1). A parental lineage can

284 include the direct parental species that hybridized to form the allopolyploid (if they are extant),

285 but also can include species closely related to the parents. For example, in Fig S1a, the true

286 network shows that the species $\mathrm{A}^{\prime}$ and $\mathrm{B}^{\prime}$ hybridized to form the polyploid lineage P. If both

287 parental species are extant and sampled, it is clear that we will recover the proper placement of

288 the hybridization event (Fig S1a). However, both of these parental species also have sister

289 species (A and B), which are part of the two parental lineages. Even with extinction of one (Fig

290 S1b) or both parental species, our method will still be able to identify the parental lineages, and

291 therefore correctly infer allopolyploidy. These two cases (Figs. S1a and b) are sometimes

292 referred to as "neopolyploidy" (Mandakova et al. 2010). Similarly, in the case of the complete

293 extinction of one parental lineage (Fig S1c; referred to as "mesopolyploidy"), our method will

294 still implicitly identify such a lineage via the placement of H1 or H2 on an internal node of the

295 singly-labeled tree. The only instance in which our method leads to an incorrect inference of the

296 type of polyploidy is in the case of the complete extinction of both parental lineages (Fig S1d;

297 sometimes referred to as "paleopolyploidy"), in which case autopolyploidy will be inferred.

298 These definitions of neo-, meso-, and paleopolyploidy are based on genealogical context alone

299 and differ from those based on cytology (e.g. Mandakova et al. 2010).

\section{Simulations}

We first checked that our modified LCA mapping algorithm counted the correct number of duplications and losses by manually reconciling a small set of 25 gene trees onto 8 MUL-trees 
and 1 singly-labeled tree to represent varying cases of gain, loss, and polyploidy. We generated our MUL-trees by starting with a single arbitrary singly-labeled species tree topology (Fig. S3a) and specifying a node as $\mathrm{H} 1$. With that node as $\mathrm{H} 1$ we tried every possible placement of node $\mathrm{H} 2$ to construct the MUL-trees (one example is shown in Fig S3b). Gene trees were made by randomly adding or removing branches from these nine trees. Our method always agreed with the expected counts for each type of event (Table S2).

Next we used gene trees simulated in the GuestTreeGen program within JPrIME (Sjöstrand et al. 2013) with varying levels of gene gain and loss and incomplete lineage sorting (ILS) to test the search feature of our algorithm. Specifically, we want to know if, given sets of gene trees simulated under conditions of allo-, auto-, or no polyploidy, our algorithm correctly identifies the type of polyploidy that has occurred and the parental lineages involved in the polyploidization event (Fig S4). To simulate scenarios with polyploidy, we started with an arbitrary MUL-tree with one clade represented twice, indicating both sub-genomes of a set of polyploid species (Fig S4a and b). JPrIME does not accept MUL-trees as input, so we added temporary marker labels to species within one of the polyploid clades. JPrIME then generated 1000 gene trees with this labeled MUL-tree as input. Gene trees were simulated under five scenarios of gain and loss rates and three scenarios of ILS, giving us 15 sets of 1000 gene trees each for each starting tree of allopolyploidy (Fig S4a), autopolyploidy (Fig S4b), and no polyploidy (Fig S4c). We removed the marker labels from the gene trees and used them as input for our algorithm, along with a singly-labeled tree in which only one of the polyploid clades is represented. We then searched for the $\mathrm{H} 1$ and $\mathrm{H} 2$ nodes that minimized the reconciliation score. For the simulations of allopolyploidy, we also gave as input to our algorithm in a separate run the 
326 alternate singly-labeled topology with the other polyploid clade represented. In all, this resulted

327 in 45 simulated datasets and 60 inferences by our algorithm. species tree topology depicted in their supplementary figures with all 27 species present (Fig. S5). We downloaded the set of 5,402 gene trees used by Marcet-Houben and Gabaldón (2015) from PhylomeDB (Phylome ID: 206). These were the main inputs to our algorithm, along with

334 the baker's yeast clade set as the H1 node (node n5 in Fig. 4a and Fig. S5). We then let our 335 algorithm search for the optimal placement of $\mathrm{H} 2$, which would allow for identification of 336 parental lineages of this polyploidy event. of labels that were either identical to or a subset of another tree. These 779 trees were removed.

339 Notung version 2.8 (Durand et al. 2006) was used to root the gene trees and to perform bootstrap 340 rearrangement with a 0.7 bootstrap threshold. Bootstrap rearrangement ensures more accurate 341 gene trees by finding the most parsimonious (with respect to duplications and losses) topology 342 around nodes with low bootstrap support by finding the lowest scoring topological ordering of

343 affected taxa. Finally, we capped our algorithm to consider at most 10 collapsed groups (Fig.

344 S1). This step cut out an additional 636 trees leaving us with a final set of 3,987 gene trees to

345 reconcile. We followed a similar procedure for the set of 963 trees containing "ohnologs" 346 obtained from Marcet-Houben and Gabaldón (2015)

347 (http://genome.crg.es/ mmarcet/yeast_hybrids/phylome_table.htm), resulting in 505 usable gene 
trees. Analysis of these trees containing homologs believed to be due to WGD yielded identical results as those of the whole dataset.

\section{Wheat}

We downloaded 55,519 gene trees from Ensembl Plants v31

\section{(ftp://ftp.ensemblgenomes.org/pub/plants/release-31/emf/ensembl-compara/homologies/)}

(Kersey et al. 2016). We also obtained the species tree that Ensembl uses for its analyses (Kersey et al. 2016). Both gene trees and species trees were pruned using Newick Utilities (Junier and Zdobnov 2010) so that they only included 10 Poaceae species, including the hexaploid bread wheat species, Triticum aestivum (Fig. S6). These species, along with all three bread wheat subgenomes, are depicted in Supplementary Figure S6. Because the D sub-genome of Triticum aestivum may be the result of homoploid hybridization between the A and $\mathrm{B}$ sub-genomes (Marcussen et al. 2014), its placement in the singly-labeled species tree is somewhat arbitrary. We chose to place the D sub-genome and its related species Aegilops tauschii as sister to the A sub-genome and its related species, Triticum urartu, in our representation of the species tree. The gene trees were filtered so they only contained between 5 and 100 tips. This left 9,147 gene trees on which to run our algorithm.

We proceeded by removing one of the three sub-genomes $(\mathrm{A}, \mathrm{B}$, or $\mathrm{D})$ from the species tree and pruning all genes that originated from that same sub-genome from the gene trees. For the remaining genes, we masked all labels that specify their sub-genome of origin. We then gave our algorithm this set of gene trees along with a singly-labeled species tree with only one of the 
two bread wheat sub-genomes represented. We allowed our algorithm to search for $\mathrm{H} 1$ and $\mathrm{H} 2$ with the expectation that the optimal MUL-tree will correctly represent both wheat sub-genomes.

\section{Results}

\section{Performance of algorithm}

Our algorithm is implemented in the software package GRAMPA (Gene-tree

Reconciliation Algorithm with MUL-trees for Polyploid Analysis; available at https://github.com/gwct/grampa). The main inputs of the program are a species tree (singlylabeled or MUL) and a set of gene trees. If a singly-labeled tree is input, possible polyploid lineages may be specified (H1 nodes; Fig. S3) along with possible placements of the second parental lineage (H2 nodes; Fig. S3). With H1 specified, GRAMPA will search for the optimal placement of the $\mathrm{H} 2$ node. If no $\mathrm{H} 1$ and $\mathrm{H} 2$ nodes are defined, GRAMPA will generate MULtrees based on all possible $\mathrm{H} 1$ and $\mathrm{H} 2$ nodes. In either case, GRAMPA will return a reconciliation score for each MUL-tree considered (as well as for the original singly-labeled tree), including the total number of duplications and losses for each individual gene tree. If a MUL-tree is input (i.e. H1 and H2 specified), GRAMPA will return a total reconciliation score for the tree and individual duplication and loss scores for the gene trees.

We checked that our modified LCA mapping algorithm counted the correct number of duplications and losses by manually reconciling a small set of 25 gene trees onto 8 MUL-trees that represent varying cases of gain and loss (Table S2). Our method always agrees with the expected counts for each type of event. GRAMPA's search method was then validated using 
larger sets of gene trees simulated using JPrIME (Sjöstrand et al. 2013) with varying rates of gene gain and loss, and varying amounts of ILS. In every simulation scenario with polyploidy that we tested GRAMPA returned the expected MUL-tree, indicating that we can correctly

394 distinguish between allo- and autopolyploidy, and that in cases of allopolyploidy we can correctly identify the parental lineages that gave rise to the polyploid species (Table S3). For example, when GRAMPA was given a set of gene trees simulated from an allopolyploidy event (Fig S4a) and a corresponding singly-labeled species tree with only one sub-genome represented, it always found the correct MUL-tree. Similarly, given a set of gene trees simulated from an autopolyploidy event (Fig S3b), GRAMPA always returns the correct autopolyploid MUL-tree. These results remain true regardless of gene gain and loss rates or levels of ILS, though with 401 increasing ILS rates we observe inflated counts of gene duplication and loss, as expected with an 402 LCA-based algorithm (Hahn 2007). We also assessed GRAMPA's performance when given a 403 singly-labeled species tree (Fig. S4c) and gene trees simulated from that species tree; in this 404 scenario no polyploidy has occurred. Reconciliation to the input singly-labeled species tree correctly resulted in the lowest score, indicating that we are able to identify when no polyploidy 406 has occurred. topologies is possible because of the penalties that naturally arise when reconciling to the 409 incorrect species topology. For instance, if allopolyploidy has occurred most gene trees should 410 have two copies of the polyploid species present, inducing a penalty of at least one duplication 411 and one loss when reconciling to a singly-labeled tree with only one copy represented (Fig. S7a).

412 This penalty is increased by one loss with each additional lineage between the two polyploid 413 clades (Fig. S7a, gene tree 1). Asymmetric gene loss between the two homoeologous sub- 
414 genomes can also provide a signal of allopolyploidy, with extra duplications and losses inferred

415 for gene trees in which homoeologs are present only in the sub-genome that is not represented in

416 the singly-labeled tree (Fig S7a, gene trees 2 and 3). Additional polyploid species make

417 asymmetric gene loss more easily detectable, increasing power to detect polyploidy. A similar

418 penalty occurs when reconciling gene trees resulting from autopolyploidy to a singly-labeled tree

419 (Fig S7b), though this penalty is less pronounced since there is only one possible singly-labeled 420 tree, making asymmetric gene loss indistinguishable.

421

423

424

425

426

427

428

429

430

431

432

433

434

\section{Analysis of baker's yeast}

We revisited the interesting case of the WGD occurring in the ancestor of Saccharomyces cerevisiae (baker's yeast), a well-known example of polyploidy (Wolfe and Shields 1997; Kellis et al. 2004). While early authors were circumspect about whether this WGD was an auto- or allopolyploid (Wolfe and Shields 1997; Kellis et al. 2004), Marcet-Houben and Gabaldón (2015) recently postulated that this clade (labeled as the "BY" clade in Fig. 4) was the result of an allopolyploidy event. These authors detected a mismatch in the timing of duplications inferred by count methods and reconciliation, as would be expected given allopolyploidy. This led to the conclusion that an ancient hybridization occurred to create an allopolyploid. This hybridization was inferred to have been between an ancestor of the ZT clade (Fig. 4) and an extinct lineage sister to the KLE, ZT, and BY clades (node n3 in Fig. 4). However, the phylogenetic methods employed by the authors to identify the parental lineages of the allopolyploid could not naturally deal with reticulation in an allopolyploidy event, and thus may have been misled by problems similar to those outlined in the Introduction. 
a)

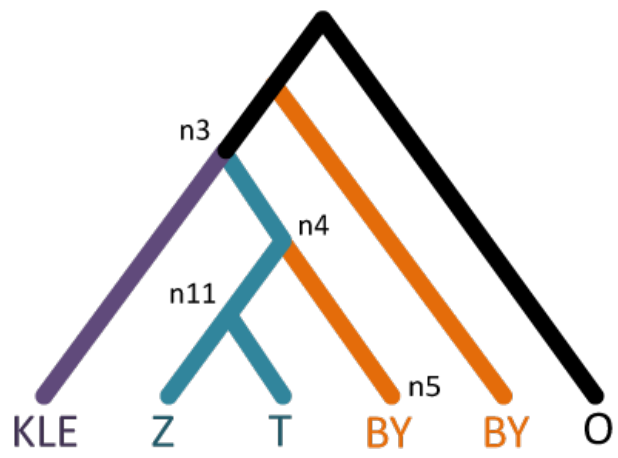
from Marcet-Houben and Gabaldón (2015) to reinvestigate the polyploid history of baker's yeast singly-labeled tree - this tree had a score of 169,031 . The fact that allopolyploidy scored much

446 lower than no polyploidy indicates that enough phylogenetic signal remains in these species to 447 differentiate the two scenarios. This signal is enhanced by the fact that there are 12 polyploid 448 species represented in the gene trees, meaning that asymmetric gene loss between sub-genomes 449 is more likely to be detected. We also compared scenarios of auto- vs. allopolyploidy. The

b)

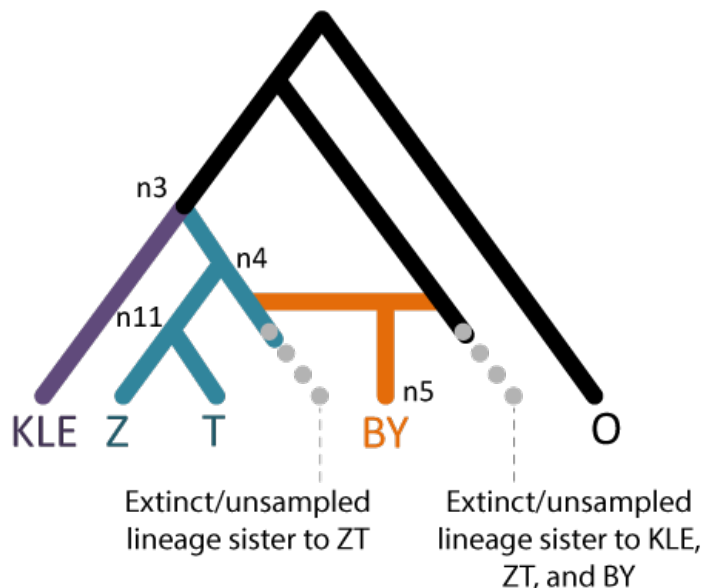
using GRAMPA. We observed that the optimal MUL-tree inferred by GRAMPA has a reconciliation score of 144,166 . We then compared this score to the scores of MUL-trees representing three alternative hypotheses. First, we wanted to confirm that we could detect the WGD by comparing the reconciliation score of the lowest scoring MUL-tree to that of the autopolyploid MUL-tree had a score of 179,636, and because the optimal allopolyploid MUL- 
451 tree has a score much lower than this, we confirm the result from Marcet-Houben and Gabaldón

452 (2015) that the modern baker's yeast clade is the result of a hybridization event.

We confirmed that GRAMPA's optimal MUL-tree also corresponds to the specific

454 allopolyploid scenario proposed by Marcet-Houben and Gabaldón (2015; Fig 4a). These results

455 suggest that the most probable parental lineages are an extinct lineage sister to the clade formed

456 by $Z$. rouxii and $T$. delbrueckii (the so-called ZT clade) and an extinct lineage sister to the KLE,

457 ZT, and the modern BY clades (Fig. 4). The next lowest scoring MUL-tree had about one

458 thousand more duplications and losses, and would have placed the second parental lineage as an 459 extinct lineage sister to only the ZT and modern BY clades. Our results therefore support the 460 claim of Marcet-Houben and Gabaldón (2015) for an allopolyploid origin of the clade including 461 baker's yeast, and further confirm their inferred parental lineages of this clade.

\section{Analysis of bread wheat}

We also applied GRAMPA to 9,147 gene trees from the clade including the hexaploid

465 species, Triticum aestivum, commonly known as bread wheat. This species is the result of three

466 hybridization events, two leading to a WGD (Petersen et al. 2006) and one resulting in a

467 homoploid hybrid species (Marcussen et al. 2014). Analysis of this clade is an especially useful

468 example to demonstrate the accuracy of GRAMPA because the relationships between sub-

469 genomes are known, and genes have been assigned to their sub-genome of origin. With genes

470 labeled according to sub-genome, standard reconciliation can be performed in an approach

471 similar to ours but with a pre-labeled MUL-tree (Altenhoff et al 2015; Bolser et al. 2015). It also 
472 presents an interesting test because the current implementation of our algorithm is only designed

473 to map one WGD per tree.

To show that GRAMPA is able to recover the correct MUL-tree for the clade including $T$.

475 aestivum and nine other Poaceae species (Fig. S6), we started by analyzing genes from two sub-

476 genomes at a time and removing all labels associating genes with sub-genomes. When we

477 allowed GRAMPA to search for the optimal MUL-tree, we recovered the one with correct sub-

478 genome relationships every time (Fig. S8, Table S5). We also investigated GRAMPA's results

479 without removing genes from any of the three sub-genomes. Interestingly, when presented with a

480 singly-labeled tree with only one sub-genome represented (Fig. S9a), GRAMPA's two lowest

481 scoring MUL-trees were those in which the other two un-represented sub-genomes were

482 identified as the $\mathrm{H} 2$ clades (Fig. S9b and c). This behavior is especially useful because it implies

483 that GRAMPA could be used to search for multiple allopolyploidy events.

\section{Discussion}

We have developed a method, GRAMPA, to accurately identify whether a polyploidy

487 event has occurred and to place it in a phylogenetic context. This allows us to identify the

488 parental lineages of the polyploid species resulting from the hybridization in the case of

489 allopolyploidy. Our method also allows us to accurately infer the number of duplications and

490 losses in a clade containing an allopolyploid. Though reconciliation methods on reticulated

491 phylogenies have been explored before (Yu et al. 2013; To and Scornavacca 2015), this is the

492 first general method that we know of that performs these types of analyses in the context of

493 polyploidy and is applicable in a wide variety of contexts. Using our method to re-analyze the 
494

495

496

497

498

499

500

501

502

503

504

505

506

507

508

509

510

511

512

513

514

515

516

neo-allopolyploid bread wheat, the results of our algorithm always align with the accepted relationships between sub-genomes. Application to the WGD in the baker's yeast clade has also confirmed both an allopolyploid origin and the parental lineages inferred previously (MarcetHouben and Gabaldón 2015). Our method works because it considers the genes duplicated during polyploidization in all possible genealogical contexts: as paralogs in the case of no polyploidy, as ohnologs in the case of autopolyploidy, and as homoeologs in the case of allopolyploidy. $\mathrm{K}_{\mathrm{s}}$ - and standard LCA-based methods fail because they do not make these distinctions.

There are cases where our method may incorrectly report that no polyploidy has occurred, even when it has. The most likely situation in which this will occur is when so many of the ohnologs or homoeologs from the WGD have been lost that the singly-labeled tree has the lowest reconciliation score. This scenario is challenging for all methods that attempt to identify WGDs (Table 1). For genes without any sort of homolog in the same genome, reconciliation to MUL-trees requires a gene loss. Because of this cost, it may seem intuitive that the point at which one can no longer infer a WGD is when more than half of all genes duplicated have returned to single-copy. As there are only $\sim 550$ homoeologs remaining in the S. cerevisiae genome (Byrne and Wolfe 2005), it may therefore be surprising that we correctly favor allopolyploidy over no polyploidy in our analysis of yeast genomes. The key factor in our statistical power to reject a history without WGD appears to be the fact that there are distinct homoeologs lost or preserved in different lineages (cf. Scannell et al. 2007). It is the sum total of these homoeologs that enable us to infer an allopolyploid history, such that the large number of polyploid species included in our analysis has helped to support this inference. We caution users of our method that any conclusions concerning the presence or absence of polyploidy is therefore 
517 dependent on many factors, including the age of the polyploidy event, the rate of duplicate gene

518 loss (and the asymmetry of loss between homoeologs), and the number of polyploid lineages

519 sampled.

521 phenomena such as incomplete lineage sorting are problems in any tree-based analysis of

522 polyploidy, and methods to both quantify and account for them have been established in other

523 contexts (e.g. Jones et al. 2013; Zwickl et al. 2014). Gene tree incongruence is also a problem

524 for reconciliation algorithms (Hahn 2007), though solutions have been proposed to deal with

525 incongruence due to ILS (e.g. Vernot et al. 2008; Rasmussen and Kellis 2012; Yu et al. 2013). In

526 the future it will be valuable to implement a similar solution for the case of reconciliation applied

527 to WGDs, or a solution based on ILS in species networks (Jones et al. 2013). Methods that do

528 not rely on gene tree reconstruction are also viable alternatives for very recent polyploidy events

529 (Roux and Pannell 2015).

The algorithm and associated software presented here should allow researchers to reexamine many published cases of polyploidy, in order to determine whether these events were

532 auto- or allopolyploidy. While many clades of plants often have multiple WGD events within

533 them, our re-analysis of the wheat data gives us confidence that our method can be expanded to

534 identify multiple polyploidy events in the same tree. For cases with only a single WGD, our

535 method provides accounting of duplication and loss, as well as the placement of these events in a 536 phylogenetic context. 
539 We thank Clara Boothby for comments on the manuscript, Marina Marcet-Houben for helpful

540 feedback regarding the yeast data, Ben Moore for information about the wheat data, and Luay

541 Nakhleh and Celine Scornavacca for pointing out important missing references. Thomas

542 Marcussen, one anonymous reviewer, and Susanne Renner also provided helpful comments. This

543 work was funded by National Science Foundation grant DBI-1564611 to MWH.

\section{References}

Adams KL, Wendel JF. 2005. Polyploidy and genome evolution in plants. Curr Opin Plant Biol. 8(2):135-141.

Altenhoff AM, Škunca N, Glover N, Train C-M, Sueki A, Piližota I, Gori K, Tomiczek B, Müller S, Redestig H, Gonnet GH, Dessimoz C. 2015. The OMA orthology database in 2015: Function predictions, better plant support, synteny view, and other improvement. Nucleic Acids Res. 43(Database issue):D240-D249.

Amores A, Force A, Yan YL, Joly L, Amemiya C, Fritz A, Ho RK, Langeland J, Prince V, Wang YL, Westerfield M, Ekker M, Postlethwait JH. 1998. Zebrafish hox clusters and vertebrate genome evolution. Science. 282(5394):1711-1714.

Barker MS, Kane NC, Matvienko M, Kozik A, Michelmore RW, Knapp SJ, Rieseberg LH. 2008. Multiple paleopolyploidizations during the evolution of the Compositae reveal parallel patterns of duplicate gene retention after millions of years. Mol Biol Evol. 25(11):2445-2455.

Barker MS, Vogel H, Schranz ME. 2009. Paleopolyploidy in the Brassicales: analyses of 
the Cleome transcriptome elucidate the history of genome duplications in Arabidopsis and other Brassicales. Genome Biol Evol. 1:391-399. autopolyploids and allopolyploids. New Phytol. 210:391-398. scale duplications in the Arabidopsis genome. Genome Res. 13(2):137-144. from age distributions of duplicate genes. The Plant Cell. ;16(7):1667-1678. Plants. Plant Cell Physiol. 56(1):e3.

Bowers JE, Chapman BA, Rong J, Paterson AH. 2003. Unravelling angiosperm genome evolution by phylogenetic analysis of chromosomal duplication events. Nature. 422(6930):433-438.

Brassac J, Blattner FR. 2015. Species-level phylogeny and polyploid relationships in Hordeum (Poeaceae) inferred by next-generation sequencing and in silico cloning of multiple nuclear loci. Syst Biol. 64(5):792-808. homology and syntenic context reveals gene fate in polyploid species. Genome Res. 15(10):1456-1461. 
early radiation of nodulating and nonnodulating legumes. Mol Biol Evol. 32(1):193-210. polyploidy in natural populations of the South American Lathyrus nervosus Lam. (Fabaceae). Plant Syst Evol. 298:761.

Crow KD, Wagner GP. 2006. What is the role of genome duplication in the evolution of complexity and diversity? Mol Biol Evol. 23(5):887-892. Widespread genome duplications throughout the history of flowering plants. Genome Res. 16(6):738-749. vertebrate. PLoS Biol. 3(10):e314. approach to gene tree reconstruction. J Comput Biol. 13(2):320-335. 
Glover NM, Redestig H, Dessimoz C. 2016. Homoeologs: What are they and how do we infer them? Trends Plant Sci. S1360-1385(16):00059-5.

Goodman M, Czelusniak J, William Moore G, Romero-Herrera AE, Matsuda G. 1979. Fitting the gene lineage into its species lineage, a parsimony strategy illustrated by cladograms constructed from globin sequences. Syst Biol. 28(2):132-163.

Guigó R, Muchnik I, Smith TF. 1996. Reconstruction of ancient molecular phylogeny. Mol Phylogenet Evol. 6(2):189-213.

Hahn MW. 2007. Bias in phylogenetic tree reconciliation methods: implications for vertebrate genome evolution. Genome Biol. 8(7):R141.

Huber KT, Moultan V. 2006. Phylogenetic networks from multi-labelled trees. J Math Biol. 52(5):613-632.

Huber KT, Oxelman B, Lott M, Moultan V. 2006. Reconstructing the evolutionary history of polyploids from multilabeled trees. Mol Biol Evol. 23(9):1784-1791. Huson DH, Rupp R, Scornavacca C. 2006. Phylogenetic networks from trees. Phylogenetic Networks, Cambridge University Press.

Jiao Y, Wickett NJ, Ayyampalayam S, Chanderbail AS, Landherr L, Ralph PE, Tomsho LP, Hu Y, Liang H, Soltis PS, SOltis DE, Clifton SW, Schlarbaum SE, Schuster SC, Ma H, Leebns-Mack J, dePamphilis CW. 2011. Ancestral polyploidy in seed plants and angiosperms. Nature. 473(7345):97-100.

Jones G, Sagitov S, Oxelman B. 2013. Statistical inference of allopolyploid species networks in the presence of incomplete lineage sorting. Syst Biol. 62(3):467-478. Junier T, Zdobnov EM. 2010. The Newick Utilities: high-throughput phylogenetic tree processing in the UNIX shell. Bioinformatics. 26:1669-1670. 
Kellis M, Birren BW, Lander ES. 2004. Proof and evolutionary analysis of ancient genome duplication in the yeast Saccharomyces cerevisiae. Nature. 428(6983):617-624.

Kersey PJ, Allen JE, Armean I, Boddu S, Bolt BJ, Carvalho-Silva D, Christensen M, Davis P, Falin LJ, Grabmueller C, Humphrey J, Kerhornou A, Khobova J, Aranganathan NK, Langridge N, Lowy E, McDowall MD, Maheswari U, Nuhn M, Kee Ong C, Overduin B, Paulini M, Pedro H, Perry E, Spudich G, Tapanari E, Walts B, Williams G, Tello-Ruiz M, Stein J, Wei S, Ware D, Bolser DM, Howe KL, Kulesha E, Lawson D, Maslen G, Staines DM. 2016. Ensembl Genomes 2016: more genomes, more complexity. Nuc Acids Res. 44(D1):D574-D580.

Kim C, Wang X, Lee TH, Jakob K, Lee GJ, Paterson AH. 2014. Comparative analysis of Miscanthus and Saccharum reveals a shared whole-genome duplication but different evolutionary fates. Plant Cell. 26(6):2420-2429.

Li Z, Baniaga AE, Sessa EB, Scascitelli M, Graham SW, Rieseberg LH, Barker MS. 2015. Early genome duplications in conifers and other seed plants. Sci Adv. 1(10):e1501084.

Linder CR, Rieseberg LH. 2004. Reconstructing patterns of reticulate evolution in plants. Am J Bot. 91(10):1700-1708.

Lott M, Spillner A, Huber KT, Petri A, Oxelman B, Moulton V. 2009. Inferring polyploid phylogenies from multiply-labeled gene trees. BMC Evol Biol. 9:216. Lynch M, Conery JS. 2000. The evolutionary fate and consequences of duplicate genes. Science. 290(5495):1151-1155.

Lynch M, Force A. 2000. The probability of duplicate gene preservation by 
subfunctionalization. Genetics. 154(1):459-473.

652

653

654

655

656

657

658

659

660

661

662

663

664

665

666

667

668

669

670

671

672

Mandáková T, Joly S, Krzywinski M, Mummenhoff K, Lysak MA. 2010. Fast

diploidization in close mesopolyploid relatives of Arabidopsis. Plant Cell.

22(7):2277-2290.

Marcussen T, Jakobsen KS, Danihelka J, Ballard HE, Blaxland K, Brysting AK,

Oxelman B. 2012. Inferring species networks from gene trees in high-polyploid

North American and Hawaiian violets (Viola, Violaceae). Syst Biol. 61(1):107-

126.

Marcussen T, Sandve SR, Heier L, Spannagl M, Pfeifer M, International Wheat Genome Sequencing Consortium, Jakobsen KS, Wulff BB, Steuernagel B, Mayer KF, Olsen OA. 2014. Ancient hybridizations among the ancestral genomes of bread wheat. Science. 345(6194):1250092.

Marcussen T, Heier L, Brysting AK, Oxelman B, Jakobsen KS. 2015. From gene trees to a dated allopolyploid network: insights from the angiosperm genus Viola (Violaceae). Syst Biol. 64(1):84-101.

Marcet-Houben M, Gabaldón T. 2015. Beyond the whole genome duplication: Phylogenetic evidence for an ancient interspecies hybridization in the baker's yeast lineage. PLoS Biol. 13(8):e1002220.

Mayrose I, Zhan SH, Rothfels CJ, Magnuson-Ford K, Barker MS, Rieseberg LH, Otto SP. 2011. Recently formed polyploid plants diversify at lower rates. Science. $333: 1257$.

Muir CD, Hahn MW. 2015. The limited contribution of reciprocal gene loss to increased 

$185: 70-86$

Ohno S. 1970. Evolution by gene duplication. Springer-Verlag

Page RDM. 1994. Maps between trees and cladistic analysis of historical associations among genes, organisms, and areas. Syst Biol. 43(1):58-77.

Petersen G, Seberg O, Yde M, Berthelsen K. 2006. Phylogenetic relationship of Triticum and Aegilops and evidence for the origin of the A, B, and D genomes of common wheat (Triticum aestivum). Mol Phylogenet Evol. 39(1):70-82.

Popp M, Oxelman B. 2001. Inferring the history of the polyploid Silene aegaea (Caryophyllaceae) using plastid and homoeologous nuclear DNA sequences. Mol Phylogenet Evol. 20(3):474-481.

Rabier C-E, Ta T, Ané C. 2014. Detecting and locating whole genome duplications on a phylogeny: A probabilistic approach. Mol Biol Evol. 31(3):750-762.

Rasmussen MD, Kellis M. 2012. Unified model of gene duplication, loss, and coalescence using a locus tree. Genome Res. 22(4):755-765.

Roux C, Pannell JR. 2015. Inferring the mode of origin of polyploidy species from nextgeneration sequence data. Mol Ecol. 24:1047-1059.

Scannell DR, Frank AC, Conant GC, Byrne KP, Woolfit M, Wolfe KH. 2007. Independent sorting-out of thousands of duplicated gene pairs in two yeast species descended from a whole-genome duplication. Proc Natl Acad Sci USA. 104(20):8397-402.

Sjöstrand J, Arvestad L, Lagergren J, Sennblad B. 2013. GenPhyloData: Realistic simulation of gene family evolution. BMC Bioinformatics. 14:209. 
Soltis PS, Soltis DE. 2009. The role of hybridization in plant speciation. Annu Rev Plant Biol. 60:561-588.

Tiley GP, Ané C, Burleigh JG. 2016 Evaluating and characterizing ancient wholegenome duplications in plants with gene count data. Genome Biol Evol. 8(4):1023-1037.

To TH, Scornavacca C. 2015. Efficient algorithms for reconciling gene trees and species networks via duplication and loss events. BMC Genomics. 16(10):S6.

Van de Peer Y. 2004. Computational approaches to unveiling ancient genome duplications. Nat Rev Genet. 5(10):752-763.

Vernot B, Stolzer M, Goldman A, Durand D. 2008. Reconciliation with non-binary species trees. J Comput Biol. 15(8):981-1006.

Werth CR, Windham MD. 1991. A model for divergent, allopatric speciation of polyploid Pteridophytes resulting from silencing of duplicate-gene expression. The American Naturalist. 137(4):515-526.

Wolfe KH. 2000. Robustness - it's not where you think it is. Nat Genet. 25(1):3-4.

Wolfe KH, Shields DC. 2007. Molecular evidence for an ancient duplication of the entire yeast genome. Nature. 287(6634):708-713.

Yu Y, Barnett RM, Nakhleh L. 2013. Parsimonious inference of hybridization in the presence of incomplete lineage sorting. Syst Biol. 62(5):738-751.

Zmasek CM, Eddy SR. 2001. A simple algorithm to infer gene duplication and speciation events on a gene tree. Bioinformatics. 17(9):821-828.

Zwickl DJ, Stein JC, Wing RA, Ware D, Sanderson MJ. 2014. Disentangling 
bioRxiv preprint doi: https://doi org/10.1101/058149; this version posted March 21, 2017. The copyright holder for this preprint (which was not certified by peer review) is the author/funder, who has granted bioRxiv a license to display the preprint in perpetuity. It is made available under aCC-BY-NC-ND 4.0 International license. 


\section{$721 \quad$ Figure Captions}

722 Figure 1. Methods to identify the placement of WGD in a phylogeny. (a) $\mathrm{K}_{\mathrm{s}}$-based methods

723 can correctly date cases of autopolyploidy. Here the peak in the distribution of $\mathrm{K}_{\mathrm{s}}$ values between

724 paralogs in a single species (shown as a density plot on the left) corresponds to the duplication

725 node in the tree. $(\boldsymbol{b}) \mathrm{K}_{\mathrm{s}}$-based methods incorrectly date cases of allopolyploidy. Here a

726 hybridization event occurred between species B and C (in blue) resulting in the allopolyploid

727 lineage that gave rise to the XYZ clade (orange). In cases like this the peak in the distribution of

$728 \mathrm{~K}_{\mathrm{s}}$ for a single species corresponds to the most recent common ancestor of the two parental

729 lineages, rather than the timing of the WGD. Similar results would be found using LCA

730 reconciliation methods discussed in the text.

Figure 2. Representation of allopolyploid clades. Given that a hybridization event occurred

733 between species $\mathrm{B}$ and $\mathrm{C}$ (in blue) resulting in the allopolyploid lineage that gave rise to the

734 XYZ clade (orange), species relationships can be represented in three ways. (a) Most species tree

735 reconstruction methods represent each species with a single label. This is not correct for

736 polyploid species as these singly-labeled species trees incorrectly represent each polyploid

737 species with a single label, showing only one of the two possible topologies. (b) Phylogenetic

738 networks correctly display the species-level phylogeny by showing hybridization events. (c)

739 Multi-labeled trees (MUL-trees) are genome-level phylogenies equivalent to networks, and

740 therefore both sub-genomes of the polyploid species are represented and it is easy to identify the

741 parental lineages involved in the hybridization event. The relative placement of hybridization in

742 the phylogeny is implicit in this representation as the point at which both sub-genomes originate. 
Figure 3. Reconciliation with a gene tree from an allopolyploid lineage. (a) A representative

747 Top: Reconciliation to a pre-defined singly-labeled species tree, with maps between gene tree nodes and species tree nodes shown (species tree nodes are labeled 1S, 2S, and 3S). In this case, genes B1 and B2 are treated as paralogs and extra duplications and losses are inferred, and the duplications are placed ancestral to the actual parental lineages (A and D) of the allopolyploid.

754 are labeled 1M, 2M, 3M, and 4M). In this case, genes B1 and B2 are treated as homoeologs and using our algorithm no extra duplications or losses are inferred.

Figure 4. The inferred MUL-tree and species network for the baker's yeast data. BY:

758 baker's yeast clade, Z: Z. rouxii, T: T. delbruekii, KLE: KLE clade, O: Outgroups. See

759 Supplementary Figure S3 for full tree and all node labels. (a) The optimal MUL-tree inferred

760 when searching for both $\mathrm{H} 1$ and $\mathrm{H} 2$. $\mathrm{H} 1$ was confirmed to be on the branch leading to the

761 baker's yeast clade (as normally represented in a species tree; specified by node n5), while H2

762 was inferred to be ancestral to the KLE, ZT, and BY clades (n3). (b) The optimal MUL-tree as a

763 network. This representation highlights the two parental lineages of the allopolyploid event: an 
764

765

766

767

768

769

770

771

772

773

774

775

776

777

778

779

780

781

782

783

784

785

extinct or unsampled lineage sister to the ZT clade, and an extinct or unsampled lineage sister the KLE, ZY, and BY clades.

\section{Supplementary Figure Captions}

Figure S1: Scenarios with various levels of extinction of parental lineages. In each panel, A' and B' have hybridized to form the polyploid clade, P. We define A' and B' as the parental species. A is more closely related to A' than B', so we say that A and A' make up one parental lineage of the polyploid (teal). Likewise, B is more closely related to B' than to A', thus making a second parental lineage (purple). (a) With no extinctions and all parental species sampled, GRAMPA will recover the correct, allopolyploid MUL-tree and correctly infer the parental lineages. (b) If a single parental species is extinct or unsampled ( $\mathrm{A}^{\prime}$ in this instance), but other species from that parental lineage are extant and sampled, GRAMPA will still infer an allopolyploid MUL-tree and correctly identify the parental lineages. This would still be the case if both parental species were extinct. (c) Even if an entire parental lineage is extinct or unsampled, GRAMPA will still infer allopolyploidy and correctly imply the second parental lineage. Only one species from one parental lineage need be present to be able to correctly infer allopolyploidy. (d) In cases where both parental lineages of an allopolyploid are entirely extinct, GRAMPA will incorrectly infer autopolyploidy with a WGD occurring between the two subgenomes of the polyploid clade (orange circle in the inferred network).

Figure S2: An example scenario of GRAMPA's heuristic speed-ups. Given the MUL-tree in (a) with two sub-genomes represented in the XYZ clade, the base algorithm will try all possible combinations of initial maps for genes from polyploid species, as in (b). If polyploid species 
group together in the gene tree, the most parsimonious initialization will always have them mapping to the same sub-genome. We collapse these into groups to reduce the number of possible maps we have to try, as in $(\boldsymbol{c})$. Furthermore, if the polyploid lineage has a sister clade in the singly-labeled species phylogeny (species A, in this case), we can look for the same pattern in the gene trees and fix the maps of any groups we find, as in $(\boldsymbol{d})$.

Figure S3: The singly-labeled species tree topology used to generate MUL-trees and gene trees for the manual reconciliations and an example MUL-tree. (a) Node H1 defines the polyploid clade made up of species $\mathrm{X}, \mathrm{Y}$, and $\mathrm{Z}$. The node ancestral to species $\mathrm{C}$ and $\mathrm{D}$ is specified as $\mathrm{H} 2$ as an example, however any other node outside of the polyploid clade can be considered as $\mathrm{H} 2$. If $\mathrm{H} 1$ and $\mathrm{H} 2$ are the same node, the resulting MUL-tree is indicative of autopolyploidy. (b) The MUL-tree generated by using the H1 and H2 nodes defined in (a).

Figure S4: The various simulation scenarios used to test GRAMPA. (a) Gene trees simulated with allopolyploidy. The allopolyploid genome topology $(\mathrm{G})$ was used as input for JPrIME. Three scenarios of ILS were considered, with the two discordant topologies also being used as input to JPrIME. Additionally, five scenarios of various gene gain-loss (G-L) rates were considered. This led to 15 sets of 1000 gene trees that were subsequently used as inputs to GRAMPA, along with both singly-labeled topologies. (b) Gene trees simulated with autopolyploidy. The autopolyploid genome topology G was used as input for JPrIME with varying scenarios of ILS and G-L. (c) Gene trees simulated with no polyploidy. The species topology (S) was used as input to JPrIME with varying scenarios of ILS and G-L. 
810 Figure S5: Yeast phylogeny. (a) The phylogeny from Marcet-Houben and Gabaldón (2015) of

81127 yeast species used in the analysis. The putative H1 node is $\mathrm{n} 5$ while the inferred $\mathrm{H} 2$ node is

812 n3. (b) A simplified version of the species tree showing only the clades and nodes of interest.

Figure S6: Wheat phylogeny. (a) The full species tree used for the bread wheat data. Common

815 bread wheat, Triticum aestivum is represented by three sub-genomes, A, B, and D. Nine other

816 closely related species were considered. (b) A simplified version of the tree showing only the

817 three sub-genomes of bread wheat (labeled A, B, and D), and the donor species of two of the 818 sub-genomes, A. tauschii and T. urartu (abbreviated AT and TU, respectively).

Figure S7: Demonstration of penalties when reconciling polyploids to singly-labeled trees.

(a) When allopolyploidy has occurred, as shown in the MUL-tree on the left, there is a much

822 higher score for reconciling to a singly-labeled tree when both homoeologous copies are retained

823 (gene tree 1) or when the copy that is not represented in the singly-labeled tree is retained while

824 the other is lost (gene tree 3), as indicated by the negative difference in reconciliation scores

$825(\Delta \mathrm{S})$. We see only relatively small penalties for reconciling to the correct MUL-tree in scenarios that favor the singly-labeled tree, such as retention of the homoeologous copy that is represented

827 in the singly-labeled tree and loss of the other copy (gene trees 2 and 4). (b) When autopolyploidy has occurred, as shown in the MUL-tree on the left, the penalties for reconciling 
830

831

832

833

834

835

836

837

838

839

840

841

842

843

844

845

846

847

848

849

850

851

tree 1). In fact, since there is only one placement of both sub-genomes, a loss of either ohnolog favors the singly-labeled tree. This means we can still distinguish autopolyploidy from no polyploidy, but have much less power to do so than with allopolyploidy (i.e. many more ohnologs must be retained to reject no polyploidy).

\section{Figure S8: Wheat polyploidy events inferred by GRAMPA when considering only 2 sub-}

genomes. In each panel, the top tree is the species tree input for GRAMPA and the bottom panel is the optimal MUL-tree inferred. The bread wheat species, Triticum aestivum, is abbreviated TA. (a) Only sub-genomes A and D are present in the gene trees and the position of TA in the input singly-labeled tree is at sub-genome A. The optimal MUL-tree shows the correct placement of sub-genome D. (b) Only sub-genomes A and B are present in the gene trees and the position of TA in the input singly-labeled tree is at sub-genome A. The optimal MUL-tree shows the correct placement of sub-genome B. (c) Only sub-genomes D and B are present in the gene trees and the position of TA in the input singly-labeled tree is at sub-genome D. The optimal MULtree shows the correct placement of sub-genome B.

\section{Figure S9: Wheat polyploidy events inferred by GRAMPA when considering all 3 sub-}

genomes. The top two scoring MUL-trees returned by GRAMPA when considering all three $T$. aestivum (TA) sub-genomes. (a) The input species tree given to GRAMPA with only subgenome A represented. (b) The best scoring MUL-tree finds sub-genome B as the other TA lineage. (d) The second best scoring MUL-tree finds sub-genome D as the other TA lineage. 